\title{
Molecular Modeling of Liquid Crystalline Self-Organization of Fullerodendrimers: Columnar to Lamellar Phase Transitions Driven by Temperature and/or Concentration Changes
}

\author{
Stavros D. Peroukidis,* Alexandros G. Vanakaras, and Demetri J. Photinos \\ Department of Materials Science, University of Patras, 26504 Patras, Greece
}

Received: June 13, 2008; Revised Manuscript Received: July 28, 2008

\begin{abstract}
The molecular cubic-block model [J. Chem. Phys. 2005, 123, 164904] is used to study a class of poly(benzyl ether) fullerodendrimers that have recently been reported to form columnar liquid crystal phases. In agreement with experiment, the model-molecules are found to self-assemble into columns which form hexagonal or rectangular lattices. The columnar cross sections are elongated in the rectangular phase. Transitions to the isotropic phase, either directly or through the intermediate formation of smectic phases, have been found. The effects of dissolving small amounts of nonbonded fullerene molecules have been explored. The results predict that the fullerene solutes restrict the range of stability of the columnar phase and may induce transitions from the columnar to the smectic or the isotropic phase.
\end{abstract}

\section{Introduction}

Supramolecular ${ }^{1}$ and/or covalent functionalization ${ }^{2}$ of $\mathrm{C}_{60}$ is an essential step toward the fabrication and manipulation of functional materials that could exploit the unique physicochemical properties of the $\mathrm{C}_{60}$ allotrope. Fuctionalization of $\mathrm{C}_{60}$ is used not only to overcome the strong tendency of the fullerenes to form aggregates ${ }^{1,2}$ but also to construct macroscopically ordered materials enabling the support of device-requisite properties. Thus, the covalent functionalization of fullerene molecules with mesomorphic dendritic addends ${ }^{3-5}$ or with mesogenic units ${ }^{3,6}$ has produced a variety of nonconventional fullerene-containing liquid crystalline (LC) compounds. These supermesogens have provided nematic mesophases with uniformly distributed fullerenes, smectic and columnar materials with the fullerene molecules forming respectively one- or twodimensional supramolecular arrays.

Recently, Deschenaux et al. ${ }^{4}$ functionalized fullerenes with poly(benzyl ether) mesogenic dendrons developed by Percec et al. ${ }^{7,8}$ The fullerene-free Percec dendrons exhibit supramolecular columnar and cubic mesophases. In these systems the flexible dendrons, depending on their generation, can adopt the shape of a disk fragment (flat-taper) or of a cone. In the ordered phases, a well defined number of these dendrons self-assemble into supramolecular disks or spheres that in turn self-organize into hexagonal columnar or cubic mesophases, respectively. The fullerene derivatives of these dendrons exhibit solely columnar mesophases. The symmetry of the columnar mesophases depends on the generation of the dendrons and may be either rectangular or hexagonal for fullerenes functionalized with second or third generation dendrons respectively.

The variety of fullerene-containing LC compounds is already broad enough to permit the deduction of certain trends and empirical design rules and also to provide testing grounds for molecular theory and computer simulation attempts toward the derivation of structure-properties relations in these systems. So far, such attempts are restricted to coarse-grained treatments of

* To whom correspondence should be addressed. E-mail: peroukid@ physics.upatras.gr. the molecular structure, either without the $\mathrm{C}_{60}$ unit, ${ }^{9-14}$ or with it, ${ }^{15-17}$ because the size of the supermesogens, dendritic or other, and their usually extensive flexibility constitute severe feasibility obstacles for reliable atomistic-detail studies of their mesomorphic behavior. However, atomistic studies have been used successfully to elucidate the conformations and packing of fullerodendrimers. ${ }^{5,18}$ On the other hand, coarse grained models of dendromesogens, endowed with the essential features (shape anisotropy, flexibility, amphipilicity) underlying liquid crystalline self-organization, ${ }^{11,12,19}$ could be very useful in rationalizing the mechanisms involved and, eventually, in suggesting new molecular design concepts. For the successful implementation of such models, their input features are normally obtained from small scale atomistic calculations. ${ }^{20,21}$

A simple approach for the coarse grained modeling of $\mathrm{C}_{60}$ supermesogens together with a tractable Onsager type molecular theory has recently been proposed by us ${ }^{15,16}$ for the study of the complex self-organization of such systems. The surprisingly good results obtained for the phase behavior and the molecular organization, including molecular self-assembly, for a variety of $\mathrm{C}_{60}$ supermesogens ${ }^{15,16}$ and of liquid crystal dendrimers ${ }^{12}$ suggest that the theoretical approach takes correctly into account the essential molecular attributes that drive the complex selforganization of these systems. The molecular features incorporated, either explicitly or implicitly, in the theory include (i) submolecular partitioning into chemically distinct units, (ii) molecular flexibility in conjunction with the existence of dominant molecular conformations and (iii) molecular directionality due to the presence of mesogenic units or due to the overall anisometric shape of the dominant molecular conformations.

In this paper, we apply our molecular theory approach to study the phase polymorphism and the molecular organization of the taper-like fullerodendrimers synthesized by Deschenaux et al. ${ }^{3-5}$ The theory reproduces successfully the supramolecular nature of the experimentally observed columnar mesophases. Furthermore, motivated by the good agreement with the experimental results, we attempt to check the predictive power 
(a)

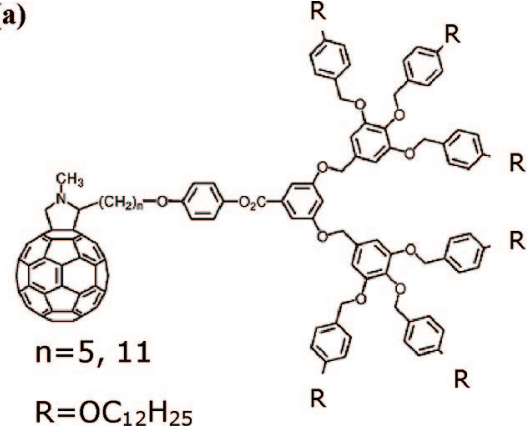

(b)

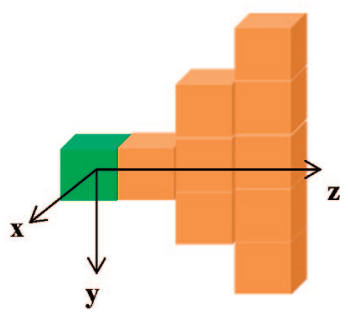

Figure 1. Chemical structure ${ }^{4}$ of a representative columnar-phase-forming fullerodendrimer (a) and its block representation used in the molecular modeling of this work (b). The dark block corresponds to the fullerene and the light ones to the dendritic components, including the aliphatic chains.

of the theory by using it to study the implications of introducing a small amount of nonbonded fullerenes into the fullerodendritic bulk phase.

\section{Theoretical Background}

The poly(benzyl ether) fullerodendrimer molecules are modeled according to the additive cubic-block model. ${ }^{15,16}$ Thus each molecule (see Figure 1) is taken to consist of 10 interacting cubic blocks, all of the same size, representing different molecular segments. One of the blocks represents the fullerene. Its size is taken equal to one fullerene diameter $(\sim 0.9 \mathrm{~nm})$. The other nine blocks correspond to the volume that is effectively available to the dendritic part, including the end chains. The relative disposition of the blocks is fixed to the triangular configuration shown in Figure 1b. This configuration is assumed to convey the shape of the statistically dominant group of conformations of the dentritic part which, aside from the pendant aliphatic chains, is expected to show limited flexibility and to be fairly flat, at least within the columnar temperature range of these systems. Each such fixed configuration of blocks, representing a fullerodendrimer of the ensemble, is restricted to translate and rotate in a cubic 3-D lattice. The lattice constants of the latter are taken to be equal to the dimensions of the elementary molecular cubic block. In all the calculations and the graphical representations of the results the unit length is identified with the side of the elementary cubic block.

The total potential energy is obtained by summing the contributions from of all possible pairs of intermolecular blocks in the ensemble. The interaction potential for a pair of blocks $b_{I}$ and $b_{J}$, belonging to molecules $I$ and $J$ respectively, is assumed to vanish if the blocks are apart by one or more lattice constants. For nearest neighbor $b_{I}-b_{J}$, the potential is likewise assumed to vanish, except if one member of the pair is a fullerene block ( $f$-block) and the other is a dendritic block ( $d$ block), in which case the pair potential is assigned a positive (repulsive) value $u^{\prime}>0$. Lastly, when the two blocks occupy the same lattice point, the pair potential is made infinitely repulsive if at least one of them is an $f$-block while if both of them are $d$-blocks the potential is taken to be finitely repulsive, with a value $u>0$ representing the relative "hardness" of the $d$-blocks. These assignments for the block-block potential correspond to physical picture of short-range interactions wherein (a) fullerenes are impenetrable to other fullerenes and to dendritic block-segments, (b) the latter exert on each other soft repulsions when their volumes overlap, and (c) a microsegregation tendency, associated with the chemically distinct character of the fullerene and dendritic components, is introduced through the disfavoring of nearest neighbor $f-d$ arrange- ments by means of a repulsive effective potential $u^{\prime}$. Further microsegregation between the aliphatic and the aromatic parts of the dendrimer can be readily incorporated into the model by introducing respective distinctions among the $d$-blocks. However, preliminary results showed that this is of minor importance in view of the primary microsegregation stemming from the distinction between $f$ - and $d$-blocks. On the other hand, as detailed in section 3.5, the distinction between aromatic and aliphatic parts was found to be necessary for an adequate description of the fullerene-free (malonate) counterparts of these systems.

Phase diagrams are calculated both, for pure fullerodendrimer systems and for binary mixtures of the latter with nonbonded fullerene units. The same intermolecular pair potential, as described above, is used irrespectively of whether the fullerenes are covalently bonded to dentritic units or not. The formulation of the free energy for the binary mixtures is based on a straightforward extension of the approach described in refs 15 and 16 and leads to the expression:

$$
\begin{gathered}
-\frac{F}{N k_{B} T} \approx \sum_{k=1}^{2}\left\{x_{k} \ln \int \mathrm{d} \varpi_{k} \zeta_{k}\left(\varpi_{k}\right)+\frac{1}{2} x_{k} \sum_{k^{\prime}=1}^{2} \times\right. \\
\left(N_{k^{\prime}}-\delta_{k, k^{\prime}}\right) \times \ln \int \mathrm{d} \varpi_{k} \int \mathrm{d} \varpi_{k^{\prime}} \rho_{k}\left(\varpi_{k}\right) \rho_{k^{\prime}}\left(\varpi_{k^{\prime}}\right) \times \\
\left.\exp \left(-U_{k k^{\prime}}\left(\varpi_{k}, \varpi_{k^{\prime}}\right) / k_{B} T\right)\right\}
\end{gathered}
$$

where $T$ is the temperature, $k_{B}$ is the Boltzmann constant, $N=$ $N_{1}+N_{2}$ is the total number of molecules, $x_{k}=N_{k} / N$ and $\zeta_{k}\left(\varpi_{k}\right)$ are the mole fraction (composition) and the variational weight function of the molecules of type $k(=1,2$, with the molecular species index $k=1$ assigned to the fullerodendrimers and $k=$ 2 to the nonbonded fullerene molecules). The variable $\varpi_{k}=$ $\left(R_{k}, \Omega_{k}\right)$ represents collectively the molecular position $R_{k}$ and orientation $\Omega_{k}$. The intermolecular potential for a pair of molecules of species $k$ and $k^{\prime}$ is denoted by $U_{k k^{\prime}}$ and $\rho_{k}\left(\varpi_{k}\right)$ is the single molecule probability distribution function of the $k$ species. The variational weight functions $\zeta_{k}\left(\varpi_{k}\right)$ in eq 1 are determined by solving the self-consistency equations resulting from the functional minimization of the free energy $F$. Clearly, in the limit $x_{1} \rightarrow 1$, the expression in eq 1 reduces to that of a pure fullerodendrimer ensemble described in ref 15 .

\section{Results and Discussion}

3.1. Phase Diagrams of Pure Fullerodendrimers. Pressuretemperature phase diagrams where calculated for the neat fullerodendrimer systems. Both the pressure and the temperature 
(a)

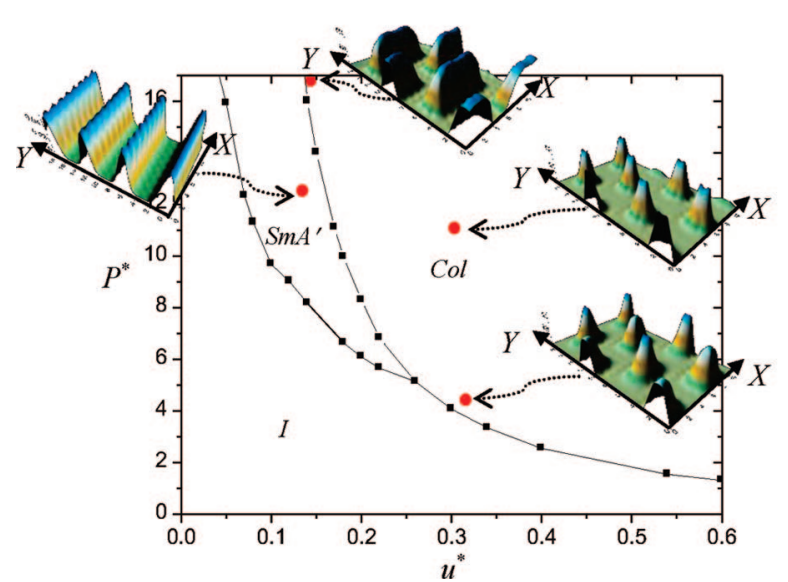

(b)

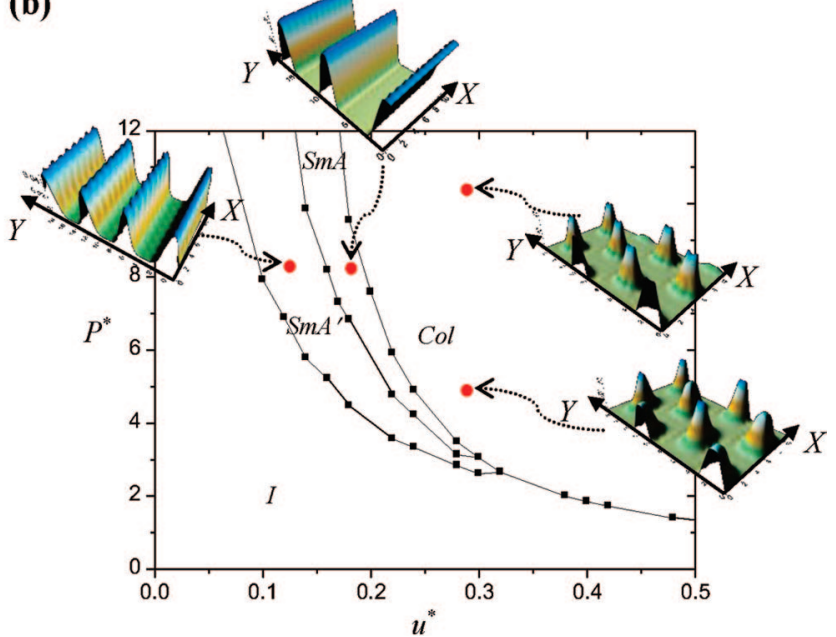

Figure 2. Calculated pressure-temperature phase diagrams of the neat fullerodendrimer system: $u^{\prime}=u$ (a) and $u^{\prime}=2 u(\mathrm{~b})$. Graphic representations of the calculated fullerene density profiles at selected points of the phase diagram are shown.

variables are scaled by the "hardness" parameter $u$ of the dendritic blocks. Accordingly, the results are plotted (see Figure 2) in terms of the reduced pressure $P^{*}=P v_{\text {block }} / u$, with $v_{\text {block }}$ denoting the volume of one elementary molecular block, and the reduced inverse temperature (equivalently, effective $d$-block hardness) $u^{*}=u / k_{B} T$. To explore the influence of microsegregation on the self-organization properties of the system, the phase diagrams were calculated for different magnitudes of the repulsive energy $u^{\prime}$ between neighboring $f-d$ block pairs. Thus, the phase diagram in Figure 2a is obtained by setting $u^{\prime}=u$ in the calculations and the one in Figure $2 b$ by doubling the microsegregation component of the interaction, i.e., by setting $u^{\prime}=2 u$. This has a general order-favoring effect, manifested by the extension of the stability range of the ordered phases to lower pressures and higher temperatures. In both cases the system exhibits an isotropic phase $(I)$, at high temperatures and low pressures, orthogonal smectic phases $\left(\operatorname{SmA}, \operatorname{Sm} A^{\prime}\right)$, at intermediate temperatures, and columnar phases $(\mathrm{Col})$ at low temperatures. The structural details of the smectic layers and of the columns are relatively sensitive to the strength of the microsegregation component and to variations of the effective hardness (inverse temperature) variable $u^{*}$. As shown below, this sensitivity is attributed to the self-assembly of the fullerodendrimers into supermolecular entities which, in turn, selforganize into layers or columns in the liquid crystalline phases. In accord with the order-favoring effect obtained by increasing $u^{\prime}$, the characteristic triple point of the isotropic-smecticcolumnar phase coexistence appears at lower temperatures and pressures in the diagram of Figure $2 b$ relative to that of Figure 2a.

For the rationalization of the phase diagrams of Figure 2 in terms of molecular packing, it is useful to note that the effective molecular volume varies considerably in the shown range of the variable $u^{*}$. Since the molecules interact via a combination of hard and soft repulsions, an appropriate measure of the effective molecular volume is provided by the average excluded volume for a pair $I, J$ of molecules $v_{e x c}=$ const $\times \int \mathrm{d} \varpi_{I} \int \mathrm{d} \varpi_{J}$ $\left[1-\exp \left(-U_{I J}\left(\varpi_{I}, \varpi_{J}\right) / k_{B} T\right)\right]$. Direct calculation shows that $v_{\text {exc }}$ monotonously increases with increasing $u^{*}$. As the molecular shape (i.e., the configuration of the blocks that form a single molecule) is fixed, the excluded volume is proportional to the effective molecular volume. Therefore, using as a reference the excluded volume $v_{\text {exc }}^{h}$ in the limit of the purely hard body interactions among all the molecular blocks (i.e., infinitely large $u$ and vanishing $u^{\prime}$ ), the volume ratio $v_{\text {exc }}\left(u^{*}\right) / v_{\text {exc }}^{h}$ represents the effective molecular volume in units of the total volume of the ten blocks that form the molecule (namely $10 \times v_{\text {block }} \approx$ $7300 \AA^{3}$ ). This volume ratio is found to increase from 0.5 at $u^{*}$ $=0.1$ to 1.2 at $u^{*}=0.8$ for the parametrization $u^{\prime}=u$ that applies to Figure 2a. The variations are even larger for $u^{\prime}=2 u$ of Figure 2b. Thus, distant $u^{*}$ regions in these phase diagrams can be viewed as describing systems of significantly different effective molecular volumes.

3.2. Structure of the Columnar Phases. The molecular organization in the columnar phase, as determined from the probability distribution function $\rho(\varpi)$ of the fullerodendrimers, is similar in both phase diagrams of Figure 2: planar selfassembled aggregates of two or more fullerodendrimers stack up to form columns which order parallel to each other in a twodimensional $c 2 \mathrm{~mm}$ rectangular lattice whose unit cell comprises two columnar cross-sections. The interior of the columns is occupied by fullerenes while the dendritic parts form the columnar periphery and fill the inter-columnar space. The distribution of the fullerenes on the plane perpendicular to the columns (the $X Y$ plane) is determined from the orientationally averaged two-dimensional density profiles $\rho_{\perp}(X, Y)=\int \mathrm{d} Z \mathrm{~d} \Omega$ $\rho(\varpi)$. Graphical representations of these profiles, calculated at different points of the phase diagrams, are shown in Figure 2. Selected profiles, together with possible arrangements of the fullerodendrimers within the columns are presented in Figure 3. It is apparent from the profiles in Figures 2 and 3 that the size and shape of the columnar cross sections, as well as the dimensions $a_{X}, a_{Y}$ of the $c 2 \mathrm{~mm}$ unit cell, vary considerably. In particular, the columnar cross sections are elongated in one direction of the lattice (defined as the $X$ direction). This elongation reflects the directional growth of the self-assembled supermolecular entities (in short, the slices) that form the columns. The number $N_{s}$ of molecules that self-assemble to form a slice is determined directly from the calculated fullerene probability distribution combined with packing considerations of the "impenetrable" fullerene blocks. The variations of the unit-cell-size with $P^{*}$ and $u^{*}$ is found to involve only the $a_{X}$ dimension of the unit-cell, with the $a_{Y}$ dimension remaining essentially constant at 10 block units. The values of the unit cell dimensions $a_{X}, a_{Y}$, together with the respective numbers $N_{s}$ of molecules per columnar slice, are given in Figure 3 for representative points of the phase diagrams. The $a_{X}$ value starts out at 6 block-lengths in the high- $u^{*}$ end of the phase diagram, 
(a)
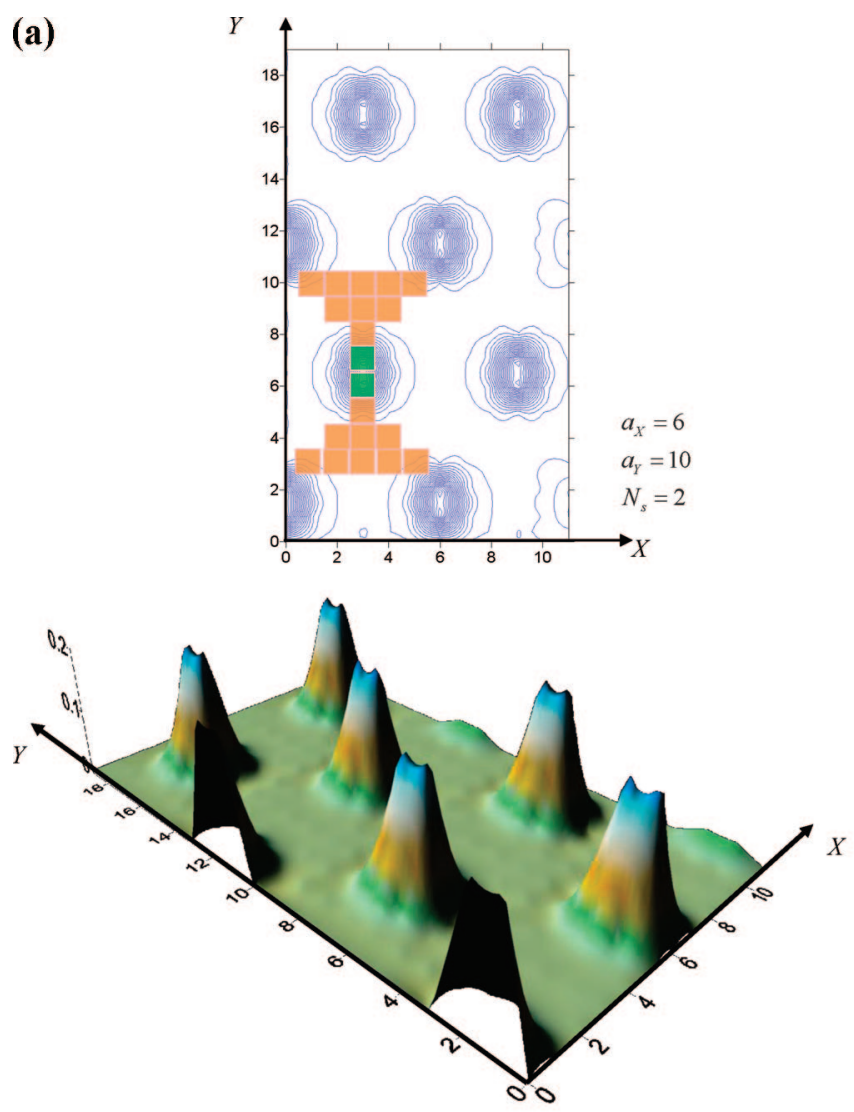

(b)
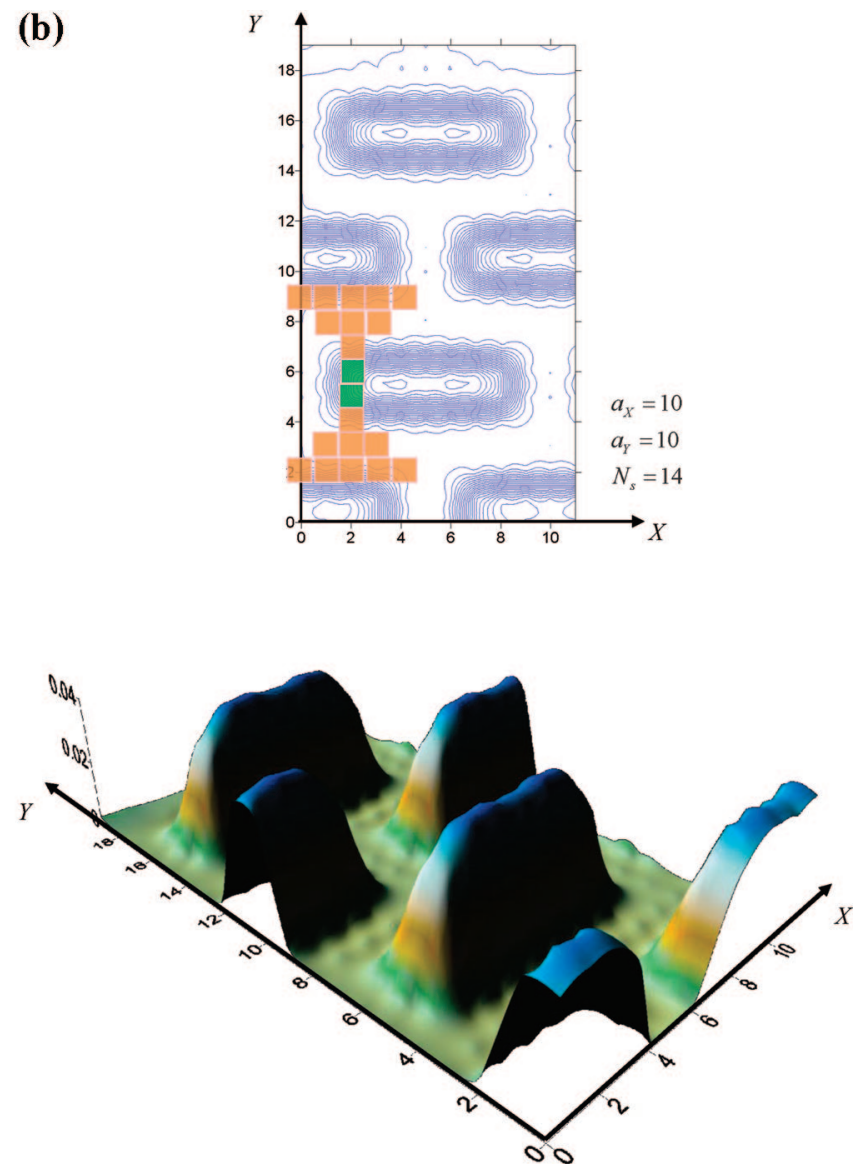

Figure 3. Density profiles and contour plots of the fullerene distribution in the $X Y$ plane of the columnar phase calculated at the points $P^{*}=$ 10.7, $u^{*}=0.28$ (a) and $P^{*}=16.5, u^{*}=0.14$ (b) of the phase diagram in Figure 2a. Distances along the $X, Y$ axes are expressed in multiples of the molecular block units $(\sim 0.9 \mathrm{~nm})$. The unit cell dimensions $a_{X}, a_{Y}$ of the 2-dimensional lattice are indicated next to the respective contour plots. Representative configurations of the fullerodendrimers, corresponding to the maximal values of the calculated probability distribution, are shown in the contour plots.

with the respective columnar slices comprising two molecules, i.e., $N_{s}=2$, and remains fairly constant on reducing $u^{*}$ except for a narrow range near the phase boundary with the smectic or the isotropic phase where $a_{X}$ and $N_{s}$ increase appreciably with decreasing $u^{*}$. On crossing the columnar to smectic phase boundary, $a_{X}$ becomes infinite. Accordingly, the phase transition can be viewed as occurring by the merging of the adjacent columns along the direction of their transverse growth (i.e., the $X$ axis) when the growth exceeds a critical value. In this picture, the $a_{Y}$ dimension of the columnar unit cell evolves into twice the smectic-layer-spacing for the $u^{\prime}=u$ system of Figure 2a, whereas for the $u^{\prime}=2 u$ system of Figure $2 \mathrm{~b} a_{Y}$ decreases to the layer spacing (eight block lengths).

Strictly, the columnar phases supported by the model are rectangular, due to the restriction to a cubic lattice. For the same reason, the changes in the unit cell dimensions $a_{X}, a_{Y}$ of the rectangular lattice can only be obtained as jumps equal to an integer number of block lengths. This precludes the possibility of a proper treatment of columnar-columnar phase transitions and the direct identification of a hexagonal columnar lattice. It would be reasonable, however, to interpret the high- $u^{*}$ columnar phases as hexagonal since the unit cell dimensions ratio $a_{X} / a_{Y}$ $=6 / 10$ obtained for these phases, within the restriction to unitblock jumps for $a_{X}, a_{Y}$, is the closest possible to the value $1 / \sqrt{ } 3$ $\approx 0.58$ characterizing the hexagonal phase. With this assignment, the increase of the ratio $a_{X} / a_{Y}$ with increasing temperature would be attributed to the appearance of a rectangular columnar phase on approaching the smectic or isotropic region of the phase diagram. Furthermore, the calculations indicate the existence of a series of columnar phases of increasing $a_{X} / a_{Y}$ ratio (i.e., elongation of the column cross section) on approaching the phase boundary. The relative thermodynamic stability of these phases is subtle and depends on the details of the model, including the imposed complete lack of molecular flexibility. The presence of these phases, however, emphasizes the elongation trend of the columnar cross sections near the hightemperature columnar phase boundary.

The above results for the columnar phase are in agreement with existing experimental observations. ${ }^{4,5}$ The $\mathrm{G}_{3} \mathrm{C}_{60}$ fullerodendrimers in ref 5 were found to form a hexagonal columnar phase in which the columnar slices comprise two molecules whereas the $\mathrm{G}_{2} \mathrm{C}_{60}$ systems and the closely related fullerodendrimers in ref 4 were found to form rectangular columnar phases of $c 2 \mathrm{~mm}$ symmetry with elongated columnar slices containing 10 to 12 molecules. With the present modeling it is straightforward to convey the generation difference between $\mathrm{G}_{2} \mathrm{C}_{60}$ and $\mathrm{G}_{3} \mathrm{C}_{60}$ by increasing the number of $d$-blocks that constitute the higher generation molecules. At a given value of $u^{*}$ this increase generates a proportional increase of the effective molecular volume. However, as noted in section 3.1, an increase of the effective molecular volume by a factor of 2 or more, can also be obtained by increasing $u^{*}$ while keeping the number of molecular blocks fixed. In this alternative, the different generations would be accommodated on the same phase diagram (i.e., calculated for same number of $d$-blocks per molecule) but at disjoint regions of the $u^{*}$ variable. The $\mathrm{G}_{3} \mathrm{C}_{60}$ molecule, having 
(a)

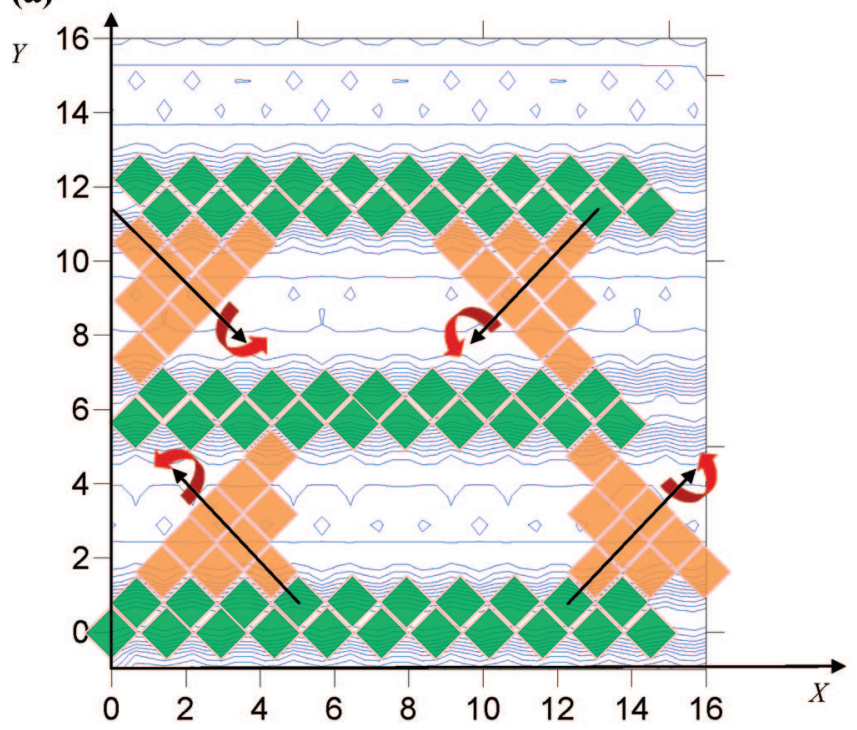

(b)

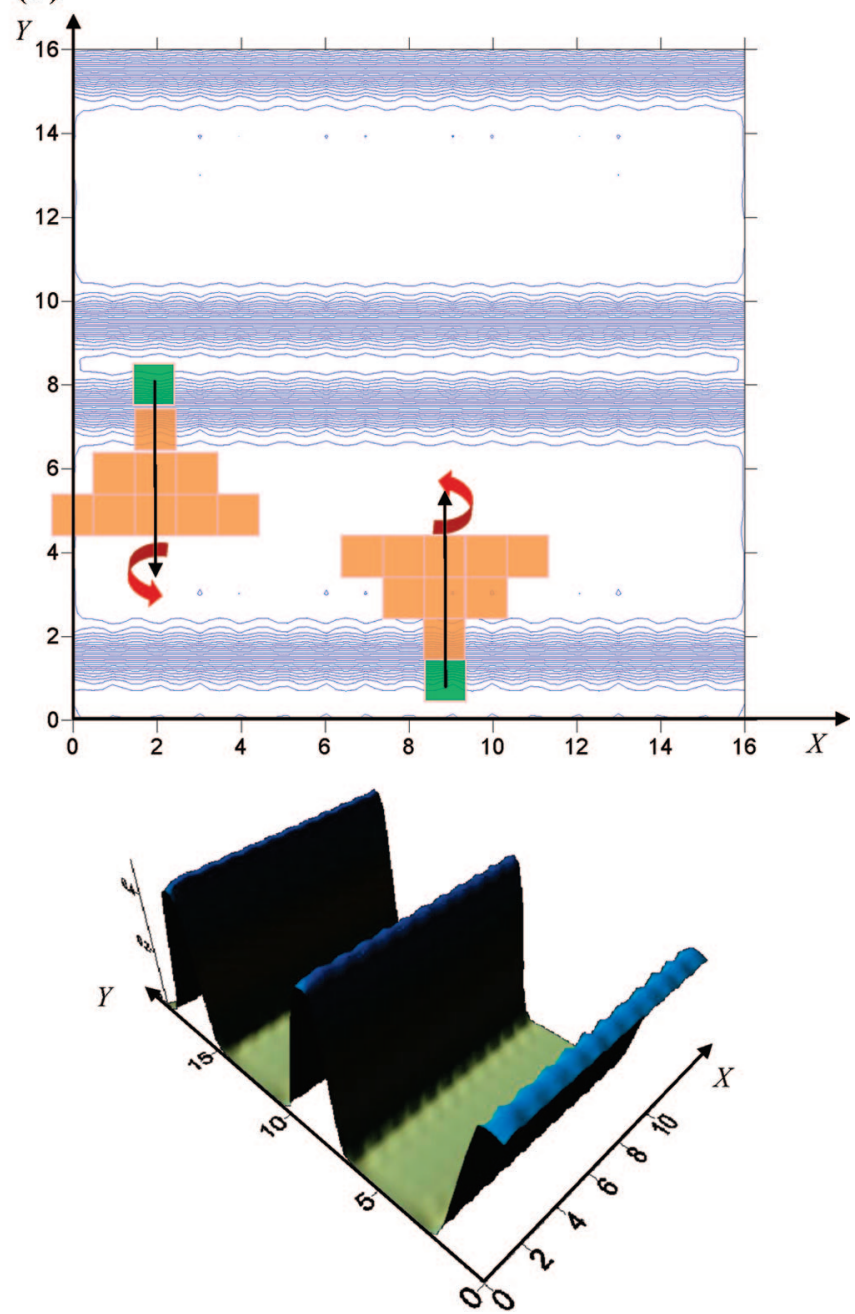

Figure 4. Calculated density profiles and contour plots of the fullerene distribution in the $X Y$ plane for the $\operatorname{Sm} A^{\prime}$ phase (a) at the points $P^{*}=$ $12.3, u^{*}=0.14$ of the phase diagram in Figure $2 \mathrm{a}$ and the $\operatorname{SmA}$ phase $(\mathrm{b})$ at the points $P^{*}=8.2, u^{*}=0.18$ of the phase diagram in Figure $2 \mathrm{~b}$. Representative configurations of the fullerodendrimers, corresponding to the maximal values of the calculated probability distribution, are inserted in the contour plots. Each of the shown configurations is 4-fold symmetric by rotation about the indicated molecular $z$ axis. Distances along the $X$, $Y$ axes are expressed in multiples of the molecular block units $(\sim 0.9 \mathrm{~nm})$.

a more developed dendritic component, would be represented by $d$-blocks whose mutual overlapping is energetically harder (i.e., corresponds to larger values of $u^{*}$ ) than the mutual overlapping of the $d$-blocks of the $\mathrm{G}_{2} \mathrm{C}_{60}$ compound, which represent a less dense dentritic component. Specifically, assigning on the phase diagram of Figure $2 \mathrm{a}$ the $\mathrm{G}_{2} \mathrm{C}_{60}$ compound to the region around $u^{*} \approx 0.14$, where the rectangular phase of elongated columnar cross sections is obtained, and assigning the $\mathrm{G}_{3} \mathrm{C}_{60}$ to the "nearly hexagonal" columnar region around $u^{*} \approx 0.54$, we obtain from the calculation of the respective excluded volumes the ratio $v_{\text {exc }}\left(u^{*}=0.54\right) / v_{\text {exc }}\left(u^{*}=0.14\right)=$ 1.8 , which coincides with the ratio of the experimentally estimated ${ }^{5}$ molecular volumes $v_{G 3 C_{60}}=8570 \AA^{3}, v_{G 2} C_{60}=4760$ $\AA^{3}$.

Within the assigned $u^{*}$ regions as above, reasonable numerical agreement is also obtained for the unit cell dimensions: The experimental value ${ }^{5}$ of the columnar diameter for the hexagonal phase of $\mathrm{G}_{3} \mathrm{C}_{60}$ is $a=4.6 \mathrm{~nm}$. This is fairly close to the calculated average columnar diameter $\bar{a}=\left(2 a_{X}+a_{Y}\right) / 4$ of the "nearly hexagonal" columnar phase of Figure 3a, which has two molecules per slice and $a_{X}=6, a_{Y}=10$ blocks, yielding $\bar{a} \approx 4.9 \mathrm{~nm}$. For the rectangular $c 2 \mathrm{~mm}$ columnar phases, the unit cell dimensions are measured in the range $a=12.8-13 \mathrm{~nm}$ and $b=8.6-8.9 \mathrm{~nm}$ for the compounds in ref 4 and $a=13.4$ $\mathrm{nm}, b=9.1 \mathrm{~nm}$ for the $\mathrm{G}_{2} \mathrm{C}_{60}$ compound in ref 5 . If the calculated, essentially constant, values of $a_{Y}$ (10 block units $9 \mathrm{~nm}$ ) are taken to correspond to the experimental $b$ values, then the respective theoretical $a_{X}$ values would be around 14 block units, which would correspond to the highly elongated columnar cross sections found near the low $u^{*}$ columnar phase boundary in the diagrams of Figure 2a.

3.3. Structure of the Smectic Phases. Structurally different smectic phases are formed by the model fullerodendrimers. The smectic phase appearing above $P^{*} \approx 5$ in the diagram of Figure 2a has a layer spacing of 5 to 6 block-lengths. Its structure consists of alternating fullerene-rich and dendrimer-rich sublayers (see Figure 4a). However, it does not correspond to any of the conventional smectics as the molecules within the layers do not present orientational ordering along a unique direction relative to the layer-normal (identified in Figure 4 with the $Y$ axis of the phase, for consistency with the diagrams of Figure $3)$. Rather there are two directions of preferred alignment of the molecular $z$ axes, at right angles to each other and directed at $45^{\circ}$ relative to the $Y$ axis of the phase, as shown in Figure 4a. Thus the layer normal $Y$ is a 2-fold rotation symmetry axis, rendering the phase an orthogonal biaxial smectic. We denote 


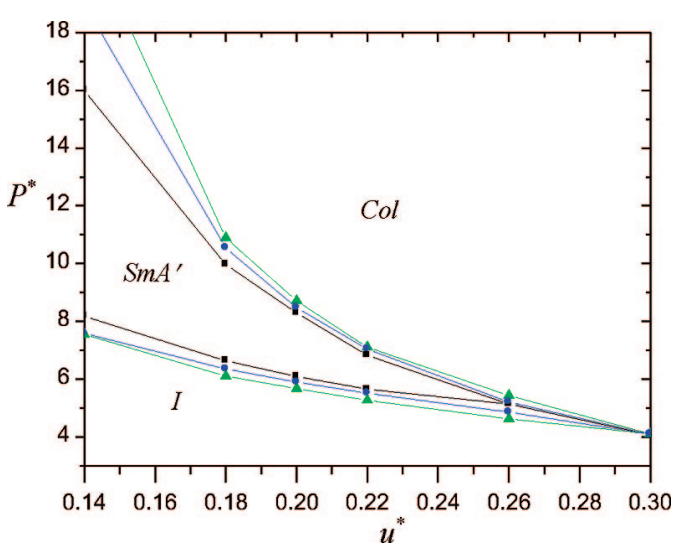

Figure 5. Calculated pressure-temperature phase diagrams for binary mixtures of fullerodendrimers with nonbonded fullerenes at concentrations $x_{2}=0.10$ (triangles) and $x_{2}=0.05$ (circles). The results are obtained with interaction parameters $u^{\prime}=u$. The phase boundaries (squares) for the neat fullerodendrimer phase $\left(x_{2}=0.0\right.$, corresponding to Figure 2a) are shown for comparison.

this phase by $S m A^{\prime}$ to distinguish it from the common $S m A$ phase in which the orientational order is defined by a single director, coincident with the layer normal. Of the two smectic phases appearing in the diagram of Figure $2 b$, the high temperature smectic has the same structure and layer spacing with the $S m A^{\prime}$ phase appearing in the diagram of Figure 2a. The lowtemperature smectic has a typical bilayer $\operatorname{SmA}$ structure; each layer consists of two nonoverlapping molecular sublayers (see Figure 4b) and the layer-spacing is 8 block units (i.e., two molecular lengths, measured along the direction of the molecular $z$ axis).

3.4. Mixtures with Nonbonded Fullerenes. The results on the pure fullerodendrimer compounds suggest a specific mechanism for the columnar to smectic transition whereby the directional growth of the columnar cross sections, driven by the microsegregation tendency of the fullerene components, leads to the merging of the columns into smectic layers. To further explore this mechanism, we have investigated the effect of adding to the system a small amount nonbonded fullerene. The anticipated outcome was a microsegragation-forced growth of the columnar cross sections beyond their critical dimensions, which would therefore destabilize the columnar phase in favor of the smectic. In other words, the increase of nonbonded fullerenes in the binary mixture would induce a columnar to smectic phase transition at constant pressure and temperature. This outcome is confirmed by the calculations, provided that the added fullerene concentrations are kept low enough to prevent a demixing transition. This is a theoretical prediction of the model as, to our knowledge, there are no experimental observations on fullerodendrimer-fullerene binary mixtures.

Figure 5 shows how the phase diagram of the model system in Figure 2a changes upon adding nonbonded fullerene molecules at concentrations $x_{2}=0.05$ and 0.10 , both assumed to be below the demixing threshold at the relevant regions of the pressure - temperature phase diagram. Clearly, the stability of the columnar phase, particularly relative to the $S m A^{\prime}$ and to a smaller extent relative to the $I$ phase, is shifted to higher pressures and lower temperatures, compared to the pure system $\left(x_{2}=0.0\right)$. On the other hand, the stability of the $S m A^{\prime}$ relative to the $I$ phase appears to expand toward lower pressures and higher temperatures. Considering that the addition of a spherical component, such as the fullerene molecule, in a binary mixture would normally favor the isotropic phase, the enhancement of the $S m A^{\prime}$ tendency found in the present case emphasizes the primary role of microsegregation in driving the isotropic to smectic phase transition in these systems.

In the columnar phase, all the fullerenes are located in the core of the columns and the dendritic parts fill the intercolumnar space. On increasing the nonbonded fullerene concentration, the unit cell is found to increase, mainly in the $X$ direction, i.e., the direction of elongation of the columnar cross section of the pure system. Thus, for example, at the point $P^{*}=13, u^{*}=0.18$ of the phase diagram the columnar unit cell dimensions change from $a_{X}=7, a_{Y}=10$ for $x_{2}=0.0$ to $a_{X}=8, a_{Y}=10$ for $x_{2}$ $=0.1$.

3.5. Self-Assembly of the Model-Supermesogens without the Fullerene Component. According to the available experimental results, ${ }^{5}$ the malonate counterparts of the $\mathrm{G}_{2} \mathrm{C}_{60}$ and $\mathrm{G}_{3} \mathrm{C}_{60}$ fullerodendrimers form hexagonal columnar phases. Thus, further testing of the block-model against experiment is provided by calculating the LC phase diagrams of the modelmolecules upon removal of the fullerene block from the molecular structure. If the other blocks in the triangular configuration of Figure 1a are left unchanged, the removal of the fullerene block annihilates the microsegregation tendency in the system. Contrary to experimental observation, the calculated phase diagrams for this case show a nematic phase separating the isotropic from the columnar phase regions. The columnar phase has unit cell dimensions $a_{X}=6, a_{Y}=6$ with two molecules per columnar slice, $N_{s}=2$, and the columns arranged in a $c 2 \mathrm{~mm}$ pattern similar to that of Figure $3 \mathrm{a}$. The presence of the nematic phase is a consequence of the complete removal of microsegregation-driving interactions. In the actual molecules, however, such interactions are present; they originate from the subdivision of each dendritic unit into aliphatic and aromatic part. This subdivision can be incorporated into the block model simply by distinguishing the five "aliphatic" blocks in the base row from the other, "aromatic", blocks of the triangular configuration in Figure 1 (with the fullerene block removed). The distinction among the two kinds of blocks is quantified, in analogy with the distinction between $f$ - and $d$-blocks, by introducing a repulsive interaction $u^{\prime \prime}$ between firstneighbor pairs of unlike blocks. In this case, calculations with $u^{\prime \prime}=u / 4$, or higher, show that the nematic phase is removed form the phase diagram for the entire temperature and pressure ranges shown in the axes of Figure 2. Of the rectangular columnar phases found, the most stable one forms a square lattice with each unit cell consisting of a single column and having dimensions $a_{X}=6, a_{Y}=6$. In this highly symmetric phase there are four molecules per columnar slice, $N_{s}=4$, arranged in a tetragonal configuration with the aliphatic blocks forming the periphery and the aromatic parts occupying the interior.

\section{Conclusions}

A highly simplified model of the generic molecular structure of fullerodendrimers bearing a poly(benzyl ether) dendron (see Figure 1) has been used: a rigid triangular configuration of partially penetrable "dendritic blocks" and an impenetrable "fullerene block" at the apex. Microsegregation is introduced simply by disfavoring energetically the nearest-neighbor disposition of unlike blocks. The introduction of further microsegregation among aliphatic and aromatic parts becomes clearly necessary for the description of the fullerene-free (malonate) systems.

Despite its extreme simplifications, the model conveys correctly the observed columnar ordering, hexagonal and rectangular, in this class of compounds and accounts for the 
elongation of the columnar cross sections in the rectangular phase. The elongation is driven by microsegregation and results from the peculiar packing of the fullerenes in the inner core of the columns: in each columnar slice the fullerene blocks form a pair of side-by-side linear arrays. The model predicts that, in addition to the experimentally observed columnar to isotropic phase transitions, phase transitions are possible to smectic mesophases as the length of these linear arrays increases beyond a critical value. The calculated phase diagrams suggest that a shifting of thermodynamic stability between smectic and columnar order can be obtained in three alternative ways: (i) by increasing the temperature, (ii) by dissolving nonbonded fullerenes in the fullerodendrimer matrix, and (iii) by increasing the mutual penetrability of the dendritic component blocks, e.g., by chemically modifying the branch density or the proportions of aliphatic to aromatic composition of the dendron. Of these three alternatives, only the last one has been to date reported experimentally, albeit in a different class, Janus-type, of fullerodendrimers ${ }^{18}$ where by chemically enhancing one of the "Janus faces" of the molecule, the equilibrium can be shifted between smectic and columnar mesomorphism.

The successful description obtained with the lattice block model of liquid crystalline fullerodendrimers, both of the type considered in this study and of various types studied previously, ${ }^{15,16}$ suggests that, in spite of the apparent molecular complexity of these systems, the underlying mechanisms that determine their mesomorphic behavior are not complex and can be rationalized in terms of molecular shape and microsegregation. This excludes, of course, systems where specific interactions (strong localized dipoles, ionic interactions, hydrogen bonding) are expected to influence significantly the molecular self-organization. Furthermore, the correct reproduction of the experimentally observed behavior makes it worthwhile to explore the theoretical predictions of columnar to smectic transitions by variation of temperature or of fullerene concentration. Notably, columnar to smectic phase transitions have not been reported, to our knowledge, for any of the fullerodendrimer types studied experimentally to date. Also notable is the fact that attempts to produce a cubic LC phase of fullerodendrimers, starting from dendritic components that do exhibit cubic phases, have not been successful., ${ }^{4,5}$ Unfortunately, the present block model is inherently not suitable for identifying reliably the molecular requirements for the stabilization of a cubic LC phase because the computational restriction to a cubic lattice artificially biases the cubic ordering.

Finally, with appropriate tuning of the block-interaction parameters $u$ and $u^{\prime}$, the results obtained for the model fullerodendrimers of this study are more generally applicable to molecular architectures combining triangular shape with distinct philicities of the submolecular regions.

Acknowledgment. S.D.P. acknowledges funding from the European Social Fund (ESF), Operational Program for Educational and Vocational Training II (EPEAEK II) through the Program IRAKLEITOS.

\section{References and Notes}

(1) Dieterich, F.; Gomex-Lopez, M. Chem. Soc. Rev. 1999, 28, 263.

(2) Diederich, F.; Thilgen, C. Science. 1996, 271, 317.

(3) Dardel, B.; Guillon, D.; Heinrich, B.; Deschenaux, R. J. Mater. Chem. 2001, 11, 2814.

(4) Lenoble, J.; Aringa, N.; Campidelli, S.; Donnio, B.; Guillon, D.; Deschenaux, R. Org. Lett. 2006, 8, 1851.

(5) Marigna, N.; Lenoble, J.; Donnio, B.; Guillon, D.; Deschenaux, R. J. Mater. Chem. 2008, 18, 1524.

(6) Sawamura, M.; Kawai, K.; Matsuo, Y.; Kanie, K.; Kato, T.; Nakamura, E. Nature. 2002, 419, 702.

(7) Percec, V.; Ahn, C.-H.; Cho, W.-D.; Jamieson, A. M.; Kim, J.; Leman, T.; Schmidt, M.; Gerle, M.; Moller, M.; Prokhorova, S. A.; Sheiko, S. S.; Cheng, S. Z. D.; Zhang, A.; Ungar, G.; Yeardley, D. J. P. J. Am. Chem. Soc. 1998, 120, 8619.

(8) Ungar, G.; Percec, V.; Holerca, M. N.; Johansson, J.; Heck, J. A. Chem. Eur. J. 2000, 6, 1258.

(9) Terzis, A. F.; Vanakaras, A. G.; Photinos, D. J. Mol. Cryst. Liq. Cryst. 1999, 330, 1761.

(10) Terzis, A. F.; Vanakaras, A. G.; Photinos, D. J. Mol. Cryst. Liq. Cryst. 2000, 352, 265.

(11) Vanakaras, A. G.; Photinos, D. J. J. Mater. Chem. 2001, 11, 2832.

(12) Vanakaras, A. G.; Photinos, D. J. J. Mater. Chem. 2001, 15, 2002.

(13) Bates, M. A. J. Chem. Phys. 2004, 120, 2026.

(14) Hughes, Z. E.; Wilson, M. R.; Stimson, L. M. Soft Matter 2005, 1, 436.

(15) Peroukidis, S. D.; Vanakaras, A. G.; Photinos, D. J. J. Chem. Phys. 2005, 123, 164904.

(16) Peroukidis, S. D.; Vanakaras, A. G.; Photinos, D. J. Soft Matter 2008, 4, 493.

(17) Sazonovas, A.; Orlandi, S.; Ricci, M.; Zannoni, C.; Gorecka, E. Chem. Phys. Lett. 2006, 430, 297.

(18) Lenoble, J.; Campidelli, S.; Maringa, N.; Donnio, B.; Guillon, D.; Yevlampieva, N.; Deschenaux, R. J. Am. Chem. Soc. 2007, 129, 9941.

(19) Tschierske, C. J. Mater. Chem. 2001, 11, 2647.

(20) Li, Y.; Lin, S.-T.; Goddard, W. A. J. Am. Chem. Soc. 2004, 126, 1872.

(21) Wilson, M. R. Int. Rev. Phys. Chem. 2005, 24, 421.

JP805214R 